\title{
Microenvironnement tumoral
}

\section{La vision du pathologiste}

$>$ Grâce aux avancées des technologies de biologie moléculaire, des progrès majeurs ont été accomplis ces dernières années dans la compréhension de la biologie tumorale. Si la cible première reste la cellule cancéreuse, le microenvironnement est clairement indissociable de cette dernière et joue un rôle primordial dans la prise en charge diagnostique, à toutes les étapes du parcours clinique et histopathologique. L'analyse du microenvironnement fait en effet partie intégrante non seulement du diagnostic histologique de tumeur (élément essentiel permettant la classification histologique des tumeurs), mais aussi de l'évaluation pronostique de la maladie, et même de la stratégie thérapeutique. Au cours des prochaines années, de nouveaux biomarqueurs pronostiques et/ou prédictifs, représentatifs du stroma tumoral, seront certainement proposés et passeront les phases de validation clinique. <

Vu par le pathologiste, le microenvironnement «normal», spécifique du tissu et de l'organe qui le contiennent, correspond, d'une part, à des éléments de la matrice extracellulaire (fibres, substance fondamentale, macromolécules et glycoprotéines), et, d'autre part, à des types cellulaires éminemment variables, identifiés et classés assez aisément dans deux grandes composantes du tissu conjonctif: le compartiment mésenchymateux (fibroblastes, myofibroblastes, adipocytes, cellules endothéliales et péricytes) et le compartiment immunitaire (lymphocytes, plasmocytes, macrophages, cellules dendritiques, mastocytes, polynucléaires). Lorsqu'une tumeur se développe, tous ces différents éléments du microenvironnement sont capables d'interagir à des degrés divers avec les cellules tumorales [1]. Cette interaction est bidirection-

Cet article fait partie du numéro thématique publié par médecine/sciences en avril 2014 et intitulé « Microenvironnement tumoral ».

nelle, puisque, d'une part, des perturbations au sein du microenvironnement peuvent avoir des effets pro-oncogéniques favorisant la tumorigenèse ou la croissance tumorale, et, d'autre part, le processus tumoral est également capable d'entraîner en retour des modifications du microenvironnement $[2-5,40](\rightarrow)$.

$\rightarrow$ Voir la Synthèse de W.H. Fridman et C. Sautès-Fridman, page 359 de ce numéro Lors du diagnostic histopathologique, le microenvironnement est décrit quotidiennement dans ce que le pathologiste nomme «stroma réaction » selon le terme consacré, et que nous désignerons par réaction stromale dans la suite de cette revue. C'est ainsi qu'en matière de pathologie tumorale sont décrits deux types principaux de réaction stromale, fibreuse ou scléro-hyaline, et inflammatoire. À ces deux éléments principaux s'ajoutent d'autres caractéristiques histologiques prises en compte par le pathologiste, telles que la présence de nécrose ou le type et l'intensité de la néovascularisation. L'exemple du cancer du sein est principalement choisi dans cette revue pour illustrer le rôle du microenvironnement tumoral dans la prise en charge diagnostique et thérapeutique.

\section{Microenvironnement et diagnostic : comment voir}

Le microenvironnement tumoral joue en fait un rôle dès les premières étapes du diagnostic de tumeur. C'est notamment le cas pour les organes superficiels (tels que le sein) où la tumeur peut être palpée par le clinicien. La sensation de dureté, qui permet d'identifier un syndrome de masse, est en effet due non pas aux cellules tumorales elles-mêmes, mais à la réaction stromale fibreuse collagénique qui les accompagne. En matière de cancer du sein, loin d'être anecdotique, il s'agit là d'un élément important dans la prise en charge puisque cette réaction stromale fibreuse révélatrice va guider successivement 

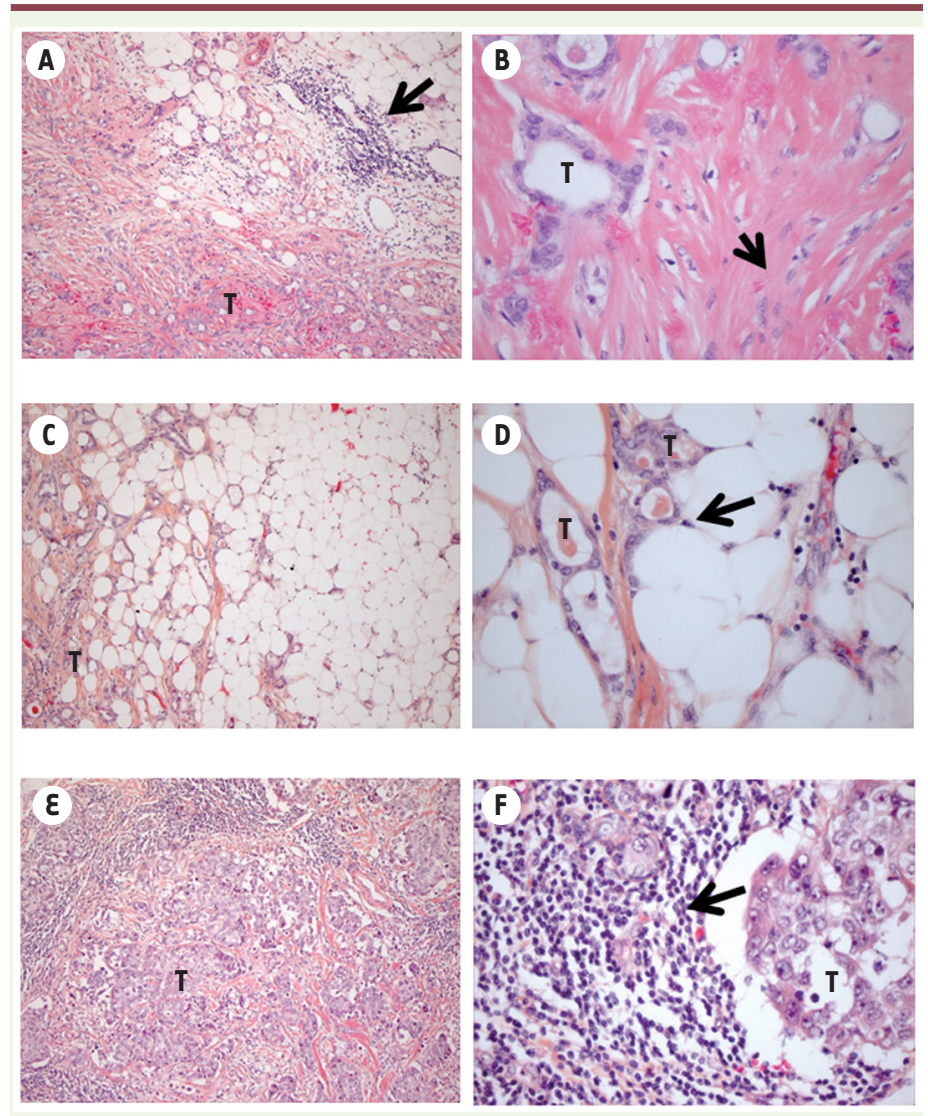

Figure 1. Exemples de réaction stromale dans les cancers du sein. A. Carcinome infiltrant sans autre spécification, montrant une réaction stromale fibreuse au centre et lymphocytaire en périphérie (hémalun-éosine, grossissement Gx10). B. Même tumeur montrant un microenvironnement fibreux et fibroblastique (hémalun-éosine, Gx40). $C$ et $D$. Périphérie d'un carcinome infiltrant de type tubuleux ( $C$, hémalun-éosine, $G \times 10$ ), montrant un contact étroit caractéristique entre glandes tumorales et adipocytes ( $D$, hémalun-éosine, Gx40). $\varepsilon$ et $F$. Carcinome infiltrant sans autre spécification, de haut grade, montrant une réaction stromale lymphocytaire marquée, intratumorale (hémalun-éosine, $\varepsilon, G \times 10$ et $F, G \times 40$ ).

le clinicien, le radiologue, le chirurgien et le pathologiste. À l'opposé, certains types de réactions stromales tumorales, telles que l'intense infiltrat lymphocytaire sans fibrose des carcinomes médullaires ou l'abondant mucus sans fibrose des tumeurs mucineuses, peuvent être responsables d'un retard de diagnostic. La tumeur, de consistance plus molle et dont les limites sont arrondies, peut en effet en imposer pour une lésion bénigne qui n'est pas cliniquement suspecte. Enfin, d'autres types de tumeurs, tels que le cancer du sein de type lobulaire, produisent peu de réaction stromale, les cellules carcinomateuses invasives s'insinuant de manière isolée dans le parenchyme mammaire, ce qui explique la difficulté de l'identification de ce type de lésion. La description de l'aspect macroscopique d'une tumeur (consistance, dureté, limites, estimation de la taille), qui est étroitement lié au microenvironnement tumoral, est ainsi l'un des premiers temps diagnostiques pour le pathologiste.
Par la suite, lors de l'examen microscopique, l'analyse du microenvironnement tumoral est également largement prise en compte parmi les arguments permettant d'aboutir au diagnostic du type histologique de tumeur. Si l'on prend l'exemple des cancers du sein, il existe de nombreux types histologiques différents, associés pour certains à un pronostic évolutif distinct $[6,41]$. La reconnaissance de ces différents types histologiques se fait non seulement sur l'aspect morphologique et l'architecture des cellules tumorales, mais aussi sur le type de réaction stromale qui les accompagne (Figure 1). Par exemple, les carcinomes tubuleux du sein, d'excellent pronostic (même meilleur que celui des carcinomes canalaires infiltrants sans autre spécification de bas grade), sont associés d'une part à une réaction stromale fibroblastique desmoplastique caractéristique, et, d'autre part, à une interaction étroite avec les adipocytes adjacents, éléments qui aident à l'établissement du diagnostic positif (Figure 1) $[6,42](\rightarrow)$.

$\rightarrow$ Voir la Synthèse de V. Laurent et al., page 398 de ce numéro

Les carcinomes de type médullaire ou avec faits médullaires, de pronostic intermédiaire, sont, eux, associés à un infiltrat inflammatoire lymphocytaire dense, étroitement mêlé aux cellules tumorales [6]. Les carcinomes micropapillaires infiltrants du sein, excessivement lymphophiles et de mauvais pronostic, sont dépourvus de la réaction stromale fibreuse classique, les cellules tumorales étant entourées de fentes de rétraction caractéristiques, liées à une polarité inversée des cellules tumorales ${ }^{1}$ [6]. Certains de ces types histologiques spéciaux de cancer du sein, tels que le carcinome infiltrant à cellules géantes ostéoclastiques, sont même exclusivement définis par la présence d'un type particulier de cellules (ici, cellules de nature macrophagique, non tumorales) dans le microenvironnement tumoral [6].

S'il est vrai que le pathologiste avait ainsi l'habitude de voir le microenvironnement tumoral et de l'intégrer de manière presque « invisible » - en tout cas automatique - dans sa démarche diagnostique, ce n'est que de façon beaucoup plus récente qu'il a été amené à le regarder directement en tant que paramètre pronostique, ou même prédictif de la réponse thérapeutique.

\footnotetext{
${ }^{1}$ Dans ce sous-type histologique, les cellules tumorales présentent en effet leur pôle apical (pôle sécrétoire) tourné vers l'extérieur des massifs tumoraux, le pôle basal se retrouvant, lui, au centre des micropapilles. L'absence de jonction cellulematrice extracellulaire au niveau du pôle apical entraîne alors une fente de rétraction, espace clair optiquement vide entre les massifs tumoraux micropapillaires et le stroma adjacent.
} 


\section{Microenvironnement et pronostic : comment regarder}

La littérature récente fournit de multiples preuves du rôle pronostique du microenvironnement tumoral, notamment dans le cancer du sein. Dans les années 2000, de nombreuses signatures moléculaires pronostiques des cancers du sein ont été proposées, mais peu portaient spécifiquement sur le microenvironnement. Cependant, de multiples publications suggèrent le rôle pronostique individuel de différents composants du microenvironnement (CD44 [récepteur de l'acide hyaluronique], $\varepsilon$-cadhérine, SPARC [secreted protein acidic and rich in cysteine ou ostéonectine], cathepsine), ce qui conduit finalement au développement de signatures multigéniques liées au stroma [7]. En 2008, Bergamaschi et al. ont développé une signature moléculaire pronostique basée sur l'expression de 278 gènes impliqués dans la biologie de la matrice extracellulaire ( $\varepsilon C M$ pour extracellular matrix) [8]. Selon cette signature, les cancers du sein peuvent être classés en quatre groupes principaux. L'un de ces groupes, nommé ECM4, est de bon pronostic et caractérisé par la surexpression d'un groupe d'inhibiteurs de protéases de la famille des serpines [8]. À l'opposé, le groupe $\mathrm{ECMl}$, de mauvais pronostic, est caractérisé par l'association d'une expression élevée d'intégrines et de métallopeptidases, et d'une expression faible de plusieurs chaînes de laminine. De façon intéressante, les tumeurs à réaction stromale fibreuse dense avec infiltrat lymphoïde auraient plutôt tendance à appartenir au groupe ECMl, tandis que les tumeurs à stroma lâche appartiendraient plutôt à la catégorie ECM4 [8]. En accord avec la taxonomie moléculaire des cancers du sein [9], on trouve plus de tumeurs de phénotype basal-like ou triple négatif (sans expression des récepteurs hormonaux ni de HER2 [human epidermal growth factor receptor 2]) dans le groupe $\mathrm{ECMI}$, alors que le groupe ECM4 est enrichi en tumeurs de type luminal A (exprimant fortement le récepteur des œstrogènes [RE]) [8].

La même année, à partir d'échantillons tissulaires microdisséqués, Finak et al. décrivent une signature pronostique basée sur 26 gènes intervenant dans la biologie du stroma [10]. Cette signature stromale permet de classer les cancers du sein en catégories de bon ou de mauvais pronostic, indépendamment des facteurs pronostiques classiques tels que le grade histologique, la taille tumorale, le statut ganglionnaire ou l'expression des récepteurs hormonaux et de HER2 [10]. Le groupe de mauvais pronostic est caractérisé par une altération de l'expression de gènes impliqués dans l'angiogenèse, la réponse à l'hypoxie, l'activation des macrophages, ou la transition épithélio-mésenchymateuse. Le groupe de bon pronostic se caractérise, quant à lui, par la surexpression de marqueurs d'une réponse immunitaire lymphocytaire $T$ de type Thl (notamment le CD8). La valeur pronostique de cette signature stromale est particulièrement puissante pour les tumeurs HER2+ [10].

Parallèlement à ces signatures issues des analyses transcriptomiques à haut débit, de multiples études se sont focalisées sur le rôle pronostique des différents compartiments cellulaires du microenvironnement tumoral (adipocytes, fibroblastes et macrophages activés, lymphocytes, cellules endothéliales) [11-18]. Mais, c'est sans aucun doute la caractérisation de la réponse immunitaire qui occupe actuellement le devant de la scène [19, 43] $(\rightarrow)$. $\rightarrow$ Voir la Synthèse de J. Galon et al., page 439 de ce numéro

L'alliance exemplaire entre pathologistes et immunologistes a en effet permis de considérables avancées dans la compréhension du rôle pronostique de la réponse immunitaire, basées notamment sur la technique d'immunohistochimie (IHC), technique de choix pour l'étude du microenvironnement tumoral. Le rôle central tenu par les lymphocytes infiltrant la tumeur (ou TIL pour tumor infiltrating lymphocytes) a été ainsi clairement démontré. Dans les cancers du sein, les TIL ont été caractérisés de façon extensive, en fonction de leur localisation (périphérique et/ou intratumorale) et de leur phénotype (B, $C D 20^{+} ; \mathrm{T} \mathrm{CD3^{+ }}, \mathrm{CD}^{+}$ou $\mathrm{CD} 8^{+}$; NK [natural killer]) [20], notamment selon les différents types histologiques (carcinome médullaire, micropapillaire) [21] ou moléculaires (basal-like, HER2, luminal B) [22-24]. Les TIL sont ainsi plus fréquemment observés dans les tumeurs $R \varepsilon^{-}$, de haut grade, de type médullaire, basal-like ou HER2+, ou chez les patientes présentant une mutation du gène de prédisposition BRCAl [21, 23, 25-27]. Sur le plan phénotypique, les TIL associés aux cancers du sein sont majoritairement de phénotype $T$, les lymphocytes $T$ cytotoxiques $C D 8^{+}$ activés (exprimant la perforine, le granzyme $B$, le ligand de Fas) attaquant principalement les cellules tumorales [21, 25-27]. De façon intéressante, l'impact pronostique des TIL (et, plus spécifiquement, la richesse en $T$ cytotoxiques $C D 8^{+}\left[C D 8^{\text {high }}\right]$ et la pauvreté en lymphocytes $T$ régulateurs $\mathrm{FOXP3}^{+}\left[\mathrm{FOXP3} 3^{\text {low }}\right]$ ) varieraient selon le type de cancer du sein, notamment selon qu'il s'agisse d'une tumeur $R \varepsilon^{+}$(rôle faible ou plutôt défavorable des TIL) ou RE' (rôle favorable des TIL) [23-26, 28]. Le rôle bénéfique de la réponse immunitaire dans les tumeurs $R \varepsilon^{-}$a été également démontré par des approches non morphologiques (transcriptomique sur broyats tumoraux) [29].

Parallèlement à la caractérisation des TIL, des études ont porté sur la vascularisation associée à la réponse immunitaire. Les HEV (high endothelial venules) sont des veinules post-capillaires spécifiquement localisées dans les zones $T$ des organes lymphoïdes secondaires. Elles permettent le recrutement des lymphocytes naîfs et des lymphocytes $\mathrm{T}$ de mémoire centrale ${ }^{2}$ à partir du sang et jouent un rôle essentiel dans la mise en place de la réponse immunitaire adaptative $[30,31]$. Les HEV sont caractérisées par un endothélium cuboïdal et l'expression abondante de molécules d'adhésion telles que les PNad (peripheral node adressins). Ces motifs de type acide sialyl-lewis ${ }^{x}$ sont impliqués dans le

${ }^{2}$ Ces cellules mémoire, $\mathrm{CCR}^{+}$(récepteur de chimiokines), expriment majoritairement le ligand CD62L et constituent une réserve pour la génération rapide de nouveaux lymphocytes T effecteurs en cas de réexposition à l'antigène. On les distingue des cellules T mémoire effectrices, qui, elles, n'expriment ni CCR7 ni CD62L. 


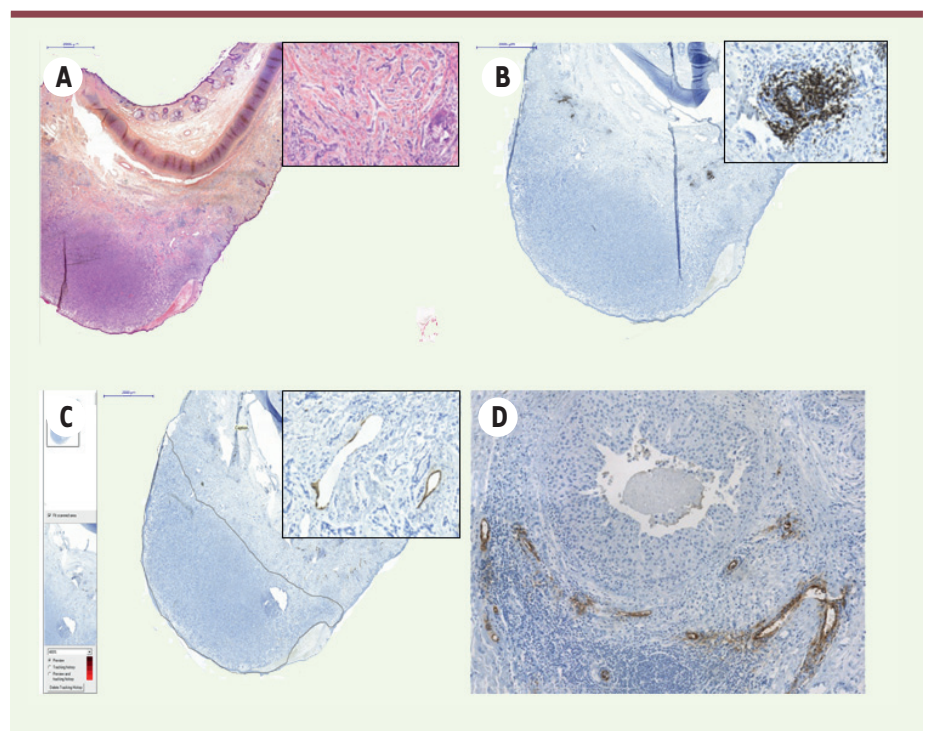

Figure 2. Étude des HEV (high endothelial venules) en pathologie tumorale - Apport des lames numériques. A. Mélanome malin du pavillon de l’oreille (hémalun-éosine, lame digitalisée). B. Immunomarquage anti-CD3, mettant en évidence un infiltrat lymphocytaire $T$ (encart) en périphérie de la tumeur, qui peut être quantifié par analyse d'image à partir de la lame digitalisée. C. La lame d'immunomarquage MECA-79 montre la présence de vaisseaux de type HEV (encart) en périphérie de la tumeur. La lame est digitalisée et la zone tumorale cerclée afin d'obtenir une surface précise en $\mathrm{mm}^{2}$. Les vaisseaux HहV sont ensuite comptés sur cette surface tumorale. D. Immunomarquage MECA-79 dans un carcinome in situ du sein, montrant la présence de vaisseaux HEV au sein de la réaction stromale lymphocytaire.

roulement (rolling) et l'attachement des lymphocytes à l'endothélium vasculaire via le récepteur CD62L (ou sélectine L) [30, 31]. Des travaux récents ont pu détecter la présence de vaisseaux de type HEV en nombre variable dans le stroma de diverses tumeurs solides humaines (mélanomes, carcinomes mammaires, ovariens, coliques et pulmonaires) par IHC à l'aide de l'anticorps MECA79 qui reconnaît spécifiquement ces vaisseaux [32,33] (Figure 2). Dans le cancer du sein, les vaisseaux HEV sont observés au sein de zones tumorales riches en lymphocytes (Figure 2D). II existe une forte corrélation entre l'infiltration lymphocytaire T et $B$ et la présence d'une forte densité de HEV dans la zone tumorale [32]. Les HEV pourraient donc jouer un rôle bénéfique dans les cancers en augmentant le recrutement de lymphocytes au sein de la tumeur.

\section{Microenvironnement et traitement : comment espérer}

Ce n'est que récemment que la littérature s'est intéressée au lien entre microenvironnement et réponse au traitement [44] $(\rightarrow)$.

Le modèle néoadjuvant (selon la séquence biopsie

$(\rightarrow)$ Voir la Synthèse de L. Borriello et Y.A. DeClerck, page 445 de ce numéro tumorale initiale, suivie d'une chimiothérapie, puis d'une exérèse chirurgicale de la tumeur) est particulièrement adapté pour étudier cette question. Dans le cancer du sein, le pourcentage de TIL est un facteur prédictif de réponse à la chimiothérapie néoadjuvante par anthracyclines/taxanes $[22,34]$. Dans une métaanalyse transcriptomique de près de 1000 cas, Ignatiadis et al. ont montré qu'un score immunitaire élevé était prédictif de réponse à la chimiothérapie néoadjuvante [35]. Une signature stromale, basée sur l'expression de 50 gènes (c'est-à-dire sur un profil d'expression génique spécifique ou métagène, dont le gène représentatif est celui qui code pour la décorine) et corrélée à la présence d'une réaction stromale fibroblastique active dans les cancers du sein $R \varepsilon^{-}$, est prédictive de résistance à une chimiothérapie néoadjuvante à base d'anthracyclines [36].

Toutes ces données suggèrent que le microenvironnement tumoral pourrait également jouer un rôle dans la prise en charge thérapeutique [37]. Les traitements anti-angiogéniques, dont la plupart ciblent le VEGF, se sont largement développés ces dernières années, en association à la chimiothérapie, dans les tumeurs solides, avec une efficacité variable selon le type de tumeur (rein, côlon, poumon, ovaire, sein), [2, 37]. La voie du FGF (fibroblast growth factor), également impliquée dans l'angiogenèse, est ciblée par de petites molécules inhibitrices du récepteur du FGF (FGFR), et plusieurs essais cliniques sont en cours dans les tumeurs solides que caractérise une altération de cette voie [37]. Le développement d'anticorps monoclonaux, par exemple contre des protéines de la matrice extracellulaire (tenascine, fibronectine), est envisagé dans différentes localisations [2, 37]. Enfin, l'immunothérapie est également activement explorée, et offre une vaste palette de molécules et d'approches différentes. On peut citer par exemple les très anciens anti-inflammatoires non stéroïdiens (inhibiteurs de COX2 [cyclooxygénase 2], testés notamment dans les cancers du sein), les inhibiteurs du TNF $\alpha$ (tumor necrosis factor), de la voie NF-KB (inhibiteur de IKK $\beta$ ), de cytokines diverses et variées, ou les vaccins $\mathrm{MUCl}$ qui induisent des réponses immunitaires cellulaire et humorale spécifiques de $\operatorname{MUCl}[2,19,37]$. La compréhension des effets secondaires, des mécanismes d'action et de résistance, et l'identification de biomarqueurs prédictifs restent des points clés du développement de ces thérapies.

\section{Perspectives pour le pathologiste}

Le matériel de choix pour l'évaluation du microenvironnement tumoral reste la coupe entière de tumeur, la technique de tissu microarray n'étant pas adaptée pour analyser l'ensemble des interactions tumeur-hôte. Les techniques d'étude les plus utilisées, telles que la 
simple coloration standard ou I'IHC, sont pratiquées de façon courante en anatomopathologie et permettent un contrôle morphologique indispensable. En effet, certains marqueurs tels que FOXP3 sont de bon pronostic lorsqu'ils sont peu représentés au niveau du contingent lymphocytaire, mais exprimés au niveau des cellules tumorales [38]. Par ailleurs, le développement des outils d'analyse d'images devrait assurer la reproductibilité et la robustesse des techniques morphologiques [20]. En complément de l'interprétation histopathologique, les outils de biologie moléculaire, qu'ils soient ou non à haut débit (RT$P C R$, transcriptomique, séquençage nouvelle génération) pourraient permettre de s'affranchir des biais de l'analyse semi-quantitative.

Le pathologiste tiendra sans nul doute un rôle primordial dans l'harmonisation et la standardisation des techniques utilisées (qu'elles soient morphologiques comme I'IHC ou moléculaires à haut débit) afin de développer des biomarqueurs rigoureux et validés, utilisables en pratique clinique. Les quantifications, localisation et caractérisation phénotypique de l'infiltrat lymphocytaire seront vraisemblablement amenées à tenir une place importante dans l'analyse des tumeurs solides, et nécessiteront l'expertise morphologique du pathologiste afin d'identifier précisément les TIL ayant un impact clinique. Enfin, il est important de rappeler que tous ces biomarqueurs potentiels, qu'ils soient pronostiques ou prédictifs, doivent passer les phases de validation selon les recommandations internationales telles que les critères EGAPP (evaluation of genomic applications in practice and prevention $)^{3}$ [39], avant toute mise en place clinique. $\diamond$

\section{SUMMARY}

Tumor microenvironment and the pathologist: looking at what we just see

Owing to the phenomenal advances in molecular technology, the past few years have seen drastic improvements in the knowledge of tumor biology. Whilst efforts are still focused on the cancer cell, it is now clear that analysis of the tumor microenvironment plays a major role in the management of cancer. Known for years as the "stroma reaction", tumor microenvironment was commonly used by pathologists as helpful criteria to establish the diagnostic of histological subtypes. More recently however, research on tumor microenvironment (and immune response in particular) has provided numerous evidences to support a leading role in the assessment of cancer prognosis, and open new avenues in treatment of cancer patients. Prognostic and/or predictive biomarkers issued from this research will have to get through the validation process required by international consensus conferences before any implementation in clinical practice. $\diamond$

\section{LIENS D'INTÉRÊT}

Les auteurs déclarent n'avoir aucun lien d'intérêt concernant les données publiées dans cet article.

\footnotetext{
${ }^{3}$ http://www.egappreviews.org/about.htm
}

\section{RÉFÉRENCES}

1. Hanahan D, Coussens LM. Accessories to the crime: functions of cells recruited to the tumor microenvironment. Cancer Cell $2012 ; 21: 309-22$.

2. Allen M, Louise Jones J. Jekyll and Hyde: the role of the microenvironment on the progression of cancer. J Pathol $2011 ; 223: 162-76$.

3. Boudreau A, van't Veer LJ, Bissell MJ. An elite hacker: breast tumors exploit the normal microenvironment program to instruct their progression and biological diversity. Cell Adh Migr 2012; 6 : 236-48.

4. Goetz JG. Tumor microenvironment indoctrination: an emerging hallmark of cancer. Cell Adh Migr 2012 ; 6 : 190-2.

5. Hanahan D, Weinberg RA. Hallmarks of cancer: the next generation. Cell $2011 ; 144: 646-74$.

6. Lakhani SR, Ellis I0, Schnitt SJ, Tan PH, van de Vijver MJ. WHO classification of tumours of the breast, $4^{\text {th }}$ ed. Lyon : IARC, 2012.

7. Chang HY, Sneddon JB, Alizadeh AA, et al. Gene expression signature of fibroblast serum response predicts human cancer progression: similarities between tumors and wounds. PLoS Biol $2004 ; 2$ : $\varepsilon 7$.

8. Bergamaschi A, Tagliabue $\varepsilon$, Sorlie T, et al. Extracellular matrix signature identifies breast cancer subgroups with different clinical outcome.J Pathol $2008 ; 214: 357-67$.

9. Perou CM, Sorlie T, Eisen MB, et al. Molecular portraits of human breast tumours. Nature $2000 ; 406: 747-52$.

10. Finak G, Bertos N, Pepin F, et al. Stromal gene expression predicts clinical outcome in breast cancer. Nat Med $2008 ; 14: 518-27$.

11. Tang $X$. Tumor-associated macrophages as potential diagnostic and prognostic biomarkers in breast cancer. Cancer Lett $2013 ; 332: 3-10$.

12. Campbell MJ, Tonlaar NY, Garwood ER, et al. Proliferating macrophages associated with high grade, hormone receptor negative breast cancer and poor clinical outcome. Breast Cancer Res Treat 2011; 128 : 703-11.

13. Van den Eynden GG, Colpaert CG, Couvelard A, et al. A fibrotic focus is a prognostic factor and a surrogate marker for hypoxia and (lymph) angiogenesis in breast cancer: review of the literature and proposal on the criteria of evaluation. Histopathology $2007 ; 51: 440-51$.

14. Servais C, Erez N. From sentinel cells to inflammatory culprits: cancerassociated fibroblasts in tumour-related inflammation. J Pathol $2013 ; 229$ : 198-207.

15. Sharma S, Sharma MC, Sarkar C. Morphology of angiogenesis in human cancer: a conceptual overview, histoprognostic perspective and significance of neoangiogenesis. Histopathology $2005 ; 46: 481-9$.

16. Hansen S, Grabau DA, Sorensen FB, et al. The prognostic value of angiogenesis by Chalkley counting in a confirmatory study design on 836 breast cancer patients. Clin Cancer Res 2000 ; $6: 139-46$.

17. Rau KM, Huang CC, Chiu T), et al. Neovascularization evaluated by CD105 correlates well with prognostic factors in breast cancers. Exp Ther Med $2012 ; 4: 231-6$.

18. Wang Yy, Lehuede C, Laurent $V$, et al. Adipose tissue and breast epithelial cells: a dangerous dynamic duo in breast cancer. Cancer Lett $2012 ; 324$ : 142-51.

19. Finn 0J. Host response in tumor diagnosis and prognosis: importance of immunologists and pathologists alliance. Exp Mol Pathol 2012 ; 93 : 315-8.

20. Kruger JM, Wemmert C, Sternberger L, et al. Combat or surveillance? Evaluation of the heterogeneous inflammatory breast cancer microenvironment. J Pathol $2013 ; 229$ : 569-78.

21. Guo X, Fan $Y$, Lang $R$, et al. Tumor infiltrating lymphocytes differ in invasive micropapillary carcinoma and medullary carcinoma of breast. Mod Pathol $2008 ; 21: 1101-7$.

22. Denkert C, Loibl S, Noske A, et al. Tumor-associated lymphocytes as an independent predictor of response to neoadjuvant chemotherapy in breast cancer. J Clin Oncol $2010 ; 28: 105-13$.

23. Loi S, Sirtaine N, Piette F, et al. Prognostic and predictive value of tumorinfiltrating lymphocytes in a phase III randomized adjuvant breast cancer trial in node-positive breast cancer comparing the addition of docetaxel to doxorubicin with doxorubicin-based chemotherapy: BIG 02-98. J Clin Oncol $2013 ; 31: 860-7$.

24. Mahmoud SM, Paish $\varepsilon C$, Powe DG, et al. Tumor-infiltrating CD8+ lymphocytes predict clinical outcome in breast cancer. J Clin Oncol $2011 ; 29: 1949-55$.

25. Baker K, Lachapelle J, Zlobec I, et al. Prognostic significance of CD8 ${ }^{+} \mathrm{T}$ lymphocytes in breast cancer depends upon both oestrogen receptor status and histological grade. Histopathology $2011 ; 58$ : 1107-16.

26. Bates GJ, Fox SB, Han C, et al. Quantification of regulatory T cells enables the identification of high-risk breast cancer patients and those at risk of late relapse. J Clin Oncol 2006 ; 24 : 5373-80. 


\section{RÉFÉRENCES}

27. Leong PP, Mohammad R, Ibrahim N, et al. Phenotyping of lymphocytes expressing regulatory and effector markers in infiltrating ductal carcinoma of the breast. Immunol Lett $2006 ; 102: 229-36$

28. Ladoire S, Mignot G, Dabakuyo S, et al. In situ immune response after neoadjuvant chemotherapy for breast cancer predicts survival. J Pathol $2011 ; 224: 389-400$.

29. Teschendorff AE, Miremadi A, Pinder SE, et al. An immune response gene expression module identifies a good prognosis subtype in estrogen receptor negative breast cancer. Genome Biol $2007 ; 8:$ R157.

30. Girard JP, Springer TA. High endothelial venules (HEVs): specialized endothelium for lymphocyte migration. Immunol Today $1995 ; 16: 449-57$.

31. Miyasaka M, Tanaka T. Lymphocyte trafficking across high endothelial venules: dogmas and enigmas. Nat Rev Immunol $2004 ; 4: 360-70$.

32. Martinet L, Garrido I, Filleron T, et al. Human solid tumors contain high endothelial venules: association with $\mathrm{T}$ - and $\mathrm{B}$-lymphocyte infiltration and favorable prognosis in breast cancer. Cancer Res $2011 ; 71: 5678-87$.

33. Michie SA, Streeter PR, Bolt PA, et al. The human peripheral lymph node vascular addressin. An inducible endothelial antigen involved in lymphocyte homing. Am J Pathol 1993; 143 : 1688-98.

34. Issa-Nummer Y, Darb-Esfahani S, Loibl S, et al. Prospective validation of immunological infiltrate for prediction of response to neoadjuvant chemotherapy in HER2-negative breast cancer: a substudy of the neoadjuvant GeparQuinto trial. PLoS One $2013 ; 8$ : e79775.

35. Ignatiadis M, Singhal SK, Desmedt C, et al. Gene modules and response to neoadjuvant chemotherapy in breast cancer subtypes: a pooled analysis. J Clin Oncol 2012 ; 30 : 1996-2004.

36. Farmer $\mathrm{P}$, Bonnefoi $\mathrm{H}$, Anderle $\mathrm{P}$, et al. A stroma-related gene signature predicts resistance to neoadjuvant chemotherapy in breast cancer. Nat Med $2009 ; 15: 68-74$.

37. Fang $\mathrm{H}$, Declerck $Y \mathrm{~A}$. Targeting the tumor microenvironment: from understanding pathways to effective clinical trials. Cancer Res $2013 ; 73: 4965-77$.
38. Ladoire S, Mignot G, Dalban C, et al. FOXP3 expression in cancer cells and anthracyclines efficacy in patients with primary breast cancer treated with adjuvant chemotherapy in the phase III UNICANCER-PACS 01 trial. Ann Oncol $2012 ; 23: 2552-61$

39. Teutsch SM, Bradley LA, Palomaki GE, et al. The evaluation of genomic applications in practice and prevention (EGAPP) initiative: methods of the EGAPP working group. Genet Med $2009 ; 11: 3-14$.

40. Fridman WH, Sautès-Fridman C. Le microenvironnement tumoral : matrice nourricière, champ de bataille et cible thérapeutique des cancers. Med Sci (Paris) $2014 ; 30: 359-65$.

41. Bertucci F, Pascal Finetti P, Cervera N, Birnbaum D. Classification pronostique du cancer du sein et profils d'expression génique sur puces à ADN. Med Sci (Paris) $2008 ; 24: 599-606$

42. Laurent $V$, Nieto $L$, Valet $P$, Muller $C$. Tissu adipeux et cancer : une association à haut risque. Med Sci (Paris) 2014 ; $30: 398-404$.

43. Galon J, Bindea G, Mlecnik B, et al. Microenvironnement immunitaire et cancer : intérêt de l'Immunoscore pour prédire l'évolution clinique. Med Sci (Paris) $2014 ; 30: 439-44$.

44. Borriello L, DeClerck YA. Le microenvironnement tumoral et la résistance thérapeutique. Med Sci (Paris) 2014 ; 30 : 445-51.
TIRÉS À PART

M. Lacroix-Triki

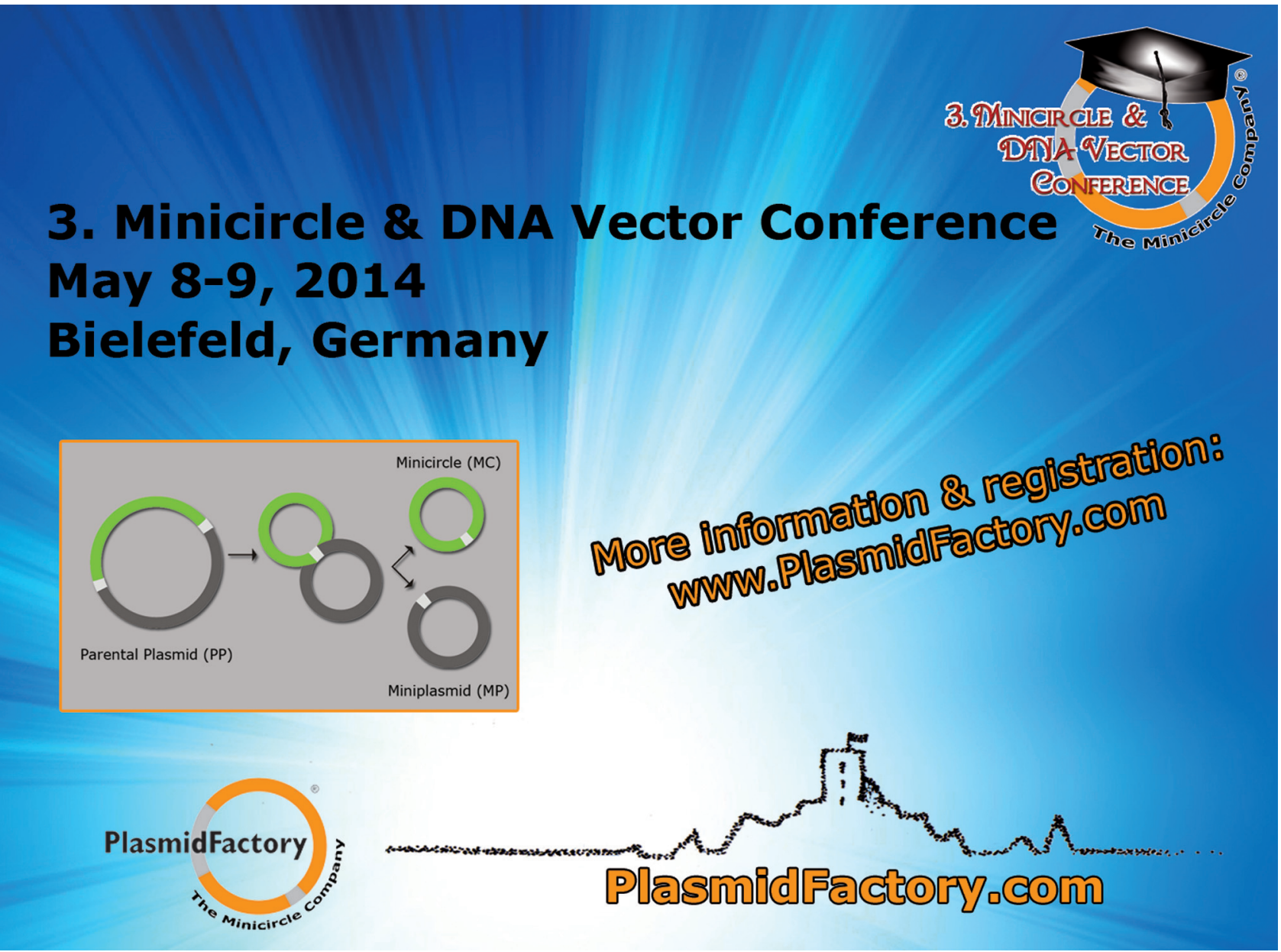

